\title{
Data from the Northern Finland 1966 Birth Cohort support an association between depression and immune function
}

\author{
Molecular Psychiatry (2003) 8, 711-712. doi:10.1038/ \\ sj.mp.4001397
}

In 1966, researchers in Finland commenced an amazing prospective epidemiological study of an unselected, genetically homogeneous general population. They studied $96 \%$ of all women $(n=12068)$, with an expected date of delivery falling between 1st January and 31st December in 1966, in the two northernmost Finnish provinces. For that 1966 Birth Cohort study, a variety of biological, socioeconomic and health-related conditions as well as living habits and family characteristics of the cohort members were collected prospectively. In a 31-year follow-up study, Timonen et al studied the association between atopy and depression in 6025 individuals (see $\mathrm{pp} \mathrm{xxx}-\mathrm{xxx}$ ). It is remarkable that $50 \%$ success rate could be achieved in a 31-year follow-up of an entire and homogeneous population.

Atopy was diagnosed by means of a combination of skin-prick tests and a clinical history of allergic symptoms and diagnoses of asthma, allergic rhinitis, atopic eczema, and/or allergic conjunctivitis. Depression was assessed by self-reported doctor-diagnosed lifetime depression and through Hopkins' Symptom Checklist-25 (HSCL-25), containing a 13-item depression subscale. Previous studies had shown that socioeconomic status, dwelling place and mother's parity were associated with atopic disorders and depression. After adjusting for those factors, logistic regression analyses showed that the risk of developing depression increased in parallel with the increasing severity of depression and, when compared with non-atopic subjects, was 3.0-4.7-fold increased in atopic females. In atopic males, the association between atopy and depression was statistically significant only in the highest depression scores, with an odds ratio of 6.3-fold.

This type of study design has considerable strengths as well as pitfalls. It includes a large number of unselected births from two provinces. Therefore, the n's are large and unbiased. Genetic stratification is not an issue as all participants have a shared genetic background. However, when studying an entire population such as this, the advantages of size are partially offset by the fact that the assessment cannot, by reasons of feasibility, be too labor intensive. The use of self-report rating scales such as the HSCL-25 conducted at one time point gives a very limited approximation of lifetime depression of cohort members, and does not provide a specific diagnosis of depression. Moreover, questionnaire studies might be biased due to interindividual differences in reporting about symptoms. In this case, in addition to HSCLscores, the authors were able to use self-reported doctor-diagnosed lifetime depression data. Moreover, biochemical data were not collected. However, a strength of the study is that skin-prick tests were used to detect atopy. These tests are one of the best objective assessments for demonstrating an IgEmediated mechanism underlying clinical atopic symptoms.

What is the relevance of these findings? First, they show that depression and atopy occur together, particularly in women. Second, they show that men and women might exhibit different substrates for depression, further documenting the biological heterogeneity of mood disorders. There are multiple possible mechanisms for an interaction between immunity and depression. Depression could be the consequence of an activated immune system. In this case, immune mediators would be the culprits for clinical symptoms as well as neuroendocrine and biochemical correlates, such as altered hypothalamic-pituitary-adrenal (HPA) axis activity and dysregulation of monoaminergic neurotransmission: cytokines can be a potent stimulus for the hypothalamic secretion of corticotropin-releasing hormone $(\mathrm{CRH})$, and they also affect serotonin and norepinephrine. Alternatively, depression could be the primary event, with possible HPA dysregulation leading to atopy. The HPA dysregulation of depression is far more complex than usually thought. For example, patients with atypical depressive features, including those seen in seasonal affective disorder, may actually have decreased HPA activity, which should predispose to hyperimmune states, such as atopy. It is likely that most depressed patients in this cohort could have atypical symptoms for two reasons: (1) depression with atypical features can be common in an outpatient, community-based sample such as this one and (2) given the extreme northern latitude of this community, it is expected that a substantial number of depression cases would be seasonal. Seasonal depression is characterized by atypical features.

A number of other factors could underlie the depression-atopy association. These factors range from histamine and lipids to shared genetic substrates. Before our imagination runs too far, it should be kept in mind that the association of two disorders could just be entirely a co-occurrence. Both conditions are common and complex; consequently, a significant overlap in one population group is not 
712 unexpected. What is particularly compelling though is that the data from the 1966 Birth Cohort increase the number of independent lines of research supporting an association between depression and altered immunity. What can be exasperating in psychiatry is the fact that we have enough lines of evidence to strongly suggest specific biological substrates, but no clearcut fundamental mechanisms of disease that can be universally accepted. That is the price of studying of some of the most complex, challenging, and fascinating phenotypes in medical science.

J Licinio and M-L Wong UCLA Laboratory for Pharmacogenomics E-mail: licinio@ucla.edu 